\title{
Supporting Information Hydration and Thermal Response Kinetics of a Cross-Linked Thermoresponsive Copolymer Film on a Hydrophobic PAN Substrate Coating Probed by In Situ Neutron Reflectivity
}

Neng Hu, ${ }^{1}$ Chen Chen, ${ }^{1}$ Ezzeldin Metwalli, ${ }^{2,}{ }^{*}$ Lorenz Bießmann, ${ }^{2}$ Christian Herold, ${ }^{2}$ Jun Fu, ${ }^{3}$ Robert Cubitt, ${ }^{4}$ Qi Zhong, ${ }^{1,2, *}$ and Peter Müller-Buschbaum ${ }^{2,5, *}$

${ }^{1}$ Key Laboratory of Advanced Textile Materials \& Manufacturing Technology, Ministry of Education, Zhejiang Sci-Tech University, 310018 Hangzhou, China

${ }^{2}$ Technische Universität München, Physik-Department, Lehrstuhl für Funktionelle Materialien, JamesFranck-Str. 1, 85748 Garching, Germany

${ }^{3}$ School of Materials Science and Engineering, Sun Yat-sen University, 135 Xingang Road West, Guangzhou 510275, China

${ }^{4}$ Institut Laue-Langevin, 6 rue Jules Horowitz, 38000 Grenoble, France

${ }^{5}$ Heinz Maier-Leibnitz Zentrum (MLZ), Technische Universität München, Lichtenbergstr. 1, 85748 Garching, Germany

\# present address: Friedrich-Alexander-Universität Erlangen-Nürnberg, Department of Condensed Matter Physics, Chair of Crystallography and Structural Physics, Staudtstr. 3, 91058 Erlangen, Germany

*Corresponding author.

muellerb@ph.tum.de, Phone +49 89289 12451, fax +49 8928912473

qi.zhong@zstu.edu.cn, Phone +86 571 86843436, fax +86 57186843436 


\section{Si substrate cleaning}

The cleaning procedure for the pre-cut Si substrate was as follows: ${ }^{1}$ To remove possible organic traces, the Si substrate was first placed in dichloromethane thermo-stated at $46{ }^{\circ} \mathrm{C}$ for $30 \mathrm{~min}$. Afterwards, it was rinsed by Millipore water for $60 \mathrm{~s}$ to remove the residual dichloromethane. Then, it was immersed into a basic solution containing water (350 mL), $\mathrm{NH}_{3}(30 \mathrm{~mL})$ and $\mathrm{H}_{2} \mathrm{O}_{2}(30 \mathrm{~mL})$ thermo-stated at $76{ }^{\circ} \mathrm{C}$ for $120 \mathrm{~min}$. After that, it was transferred into Millipore water and dried by nitrogen flow before spincoating.

\section{White light interferometry (WLI) measurements}

The film was placed horizontally in a customized aluminum chamber. The incident white-light beam (wavelength of 400-1100 nm) passed through the glass windows onto the sample. As the incident beam had multiple reflections between the different parallel surfaces, the multiple beams interfered with each other, which resulted in a net transmission and reflection amplitude based on the wavelength of the incident beam. Thus, a series of fringes as a function of wavelength was obtained. By fitting the fringes with a model containing a single layer structure, the film thickness and the refractive index $(n)$ were obtained. ${ }^{2}$

For the film hydration, $8 \mathrm{~mL} \mathrm{D}_{2} \mathrm{O}$ was injected into the aluminum chamber to install a moderate $\mathrm{D}_{2} \mathrm{O}$ vapor atmosphere (relative humidity, $\mathrm{RH}=90 \%$ ). The measurement was performed every $30 \mathrm{~s}$ to monitor the evolution of film thickness at $18{ }^{\circ} \mathrm{C}$. After the hydration process reached an equilibrium, the external temperature was gradually 
increased from 18 to $50{ }^{\circ} \mathrm{C}$ in steps of $2{ }^{\circ} \mathrm{C}$ to study the transition of the cross-linked $\mathrm{P}\left(\mathrm{MEO}_{2} \mathrm{MA}-\mathrm{co}-\mathrm{OEGMA}_{300}\right)$ film on the PAN substrate coating. To ensure the crosslinked film was reaching an equilibrium state, the film thickness was recorded $20 \mathrm{~min}$ after each increase of temperature. The temperature in the chamber was controlled by a Julabo F12 MC thermal bath (JULABO Labortechnik GmbH, Seelbach, Germany).

a)

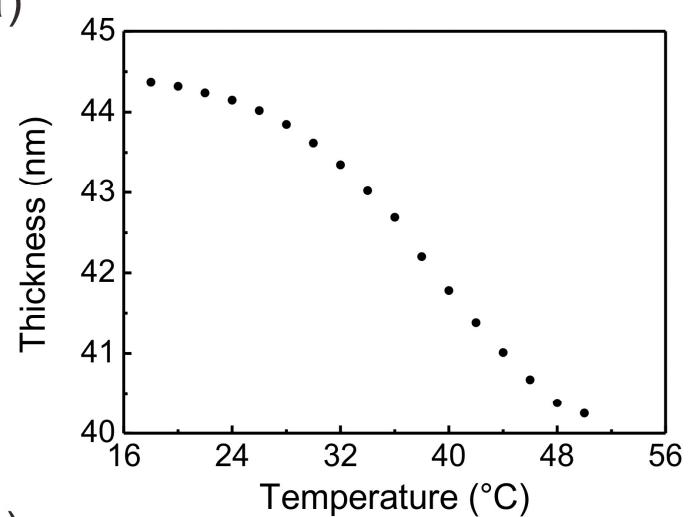

b)

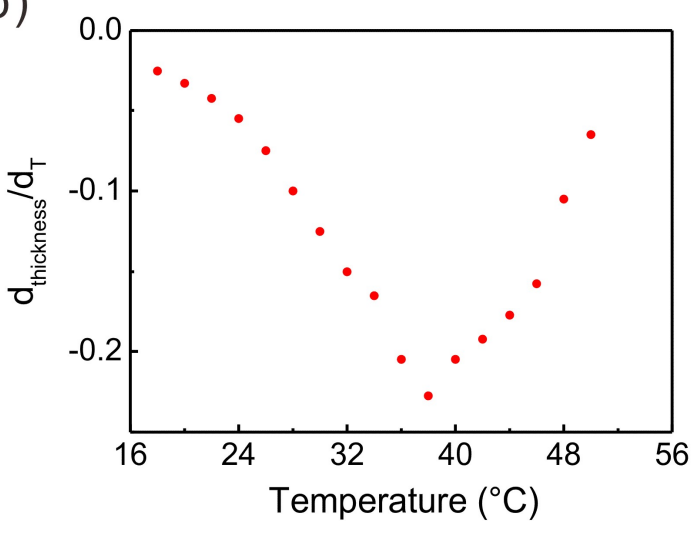

Figure S1. Temperature-dependent evolution of (a) the film thickness and (b) the first derivative of film thickness over temperature. 
a)

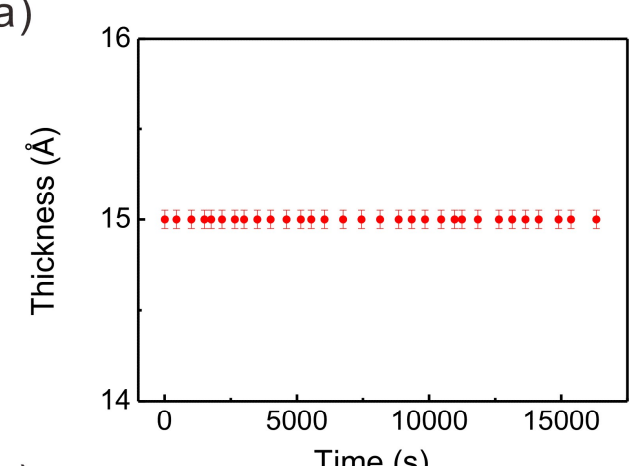

c)

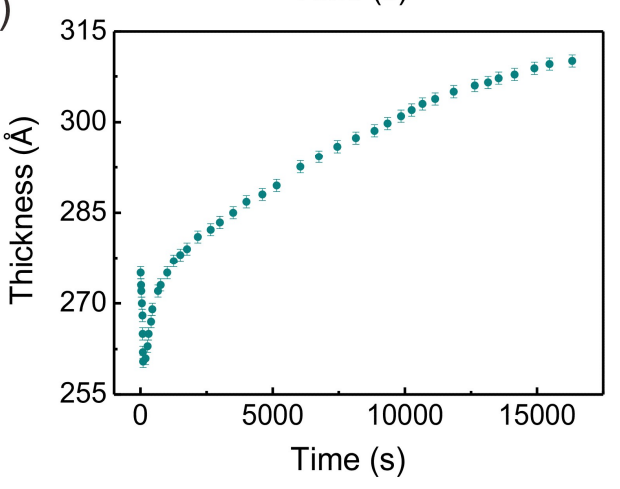

b)

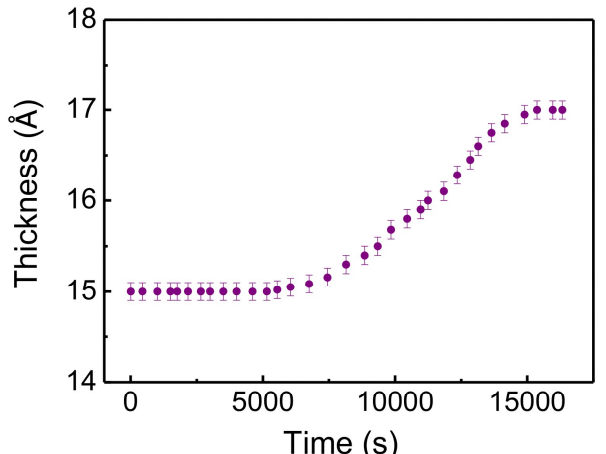

d)

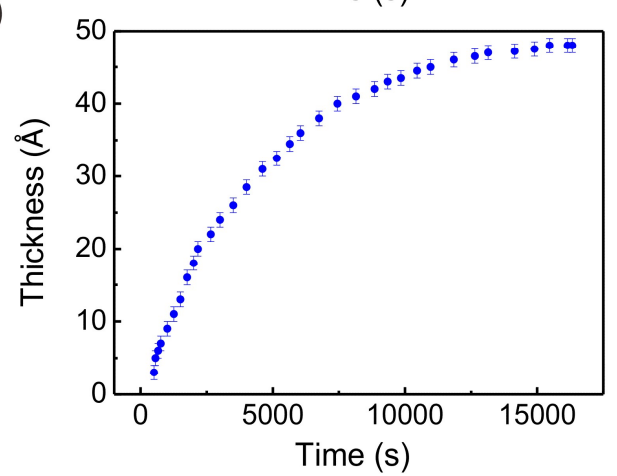

Figure S2. Thickness evolution in (a) PAN substrate coating (red), (b) substrate-near layer (purple), (c) main layer (dark green) and (d) vapor-near layer (blue) during the hydration in $\mathrm{D}_{2} \mathrm{O}$ vapor atmosphere at $23{ }^{\circ} \mathrm{C}$.

Table S1. Obtained fit parameters of vapor-near layer and main layer in the cross-linked film during hydration.

\begin{tabular}{cccc}
\hline & $\tau / \mathrm{s}$ & $\mathrm{B}$ & $\varphi\left(\mathrm{D}_{2} \mathrm{O}\right)_{\infty} / \%$ \\
\hline vapor-near layer & $4750 \pm 20$ & $0.84 \pm 0.02$ & $24.2 \pm 0.5$ \\
main layer & $5300 \pm 20$ & $0.83 \pm 0.02$ & $32.1 \pm 0.5$ \\
\hline
\end{tabular}


a)

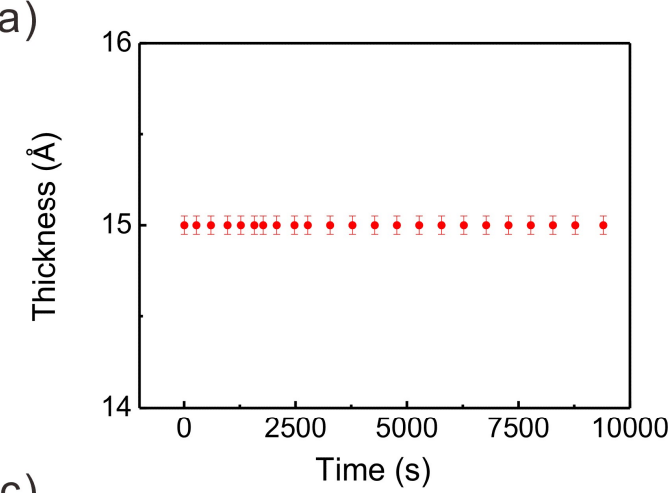

c)

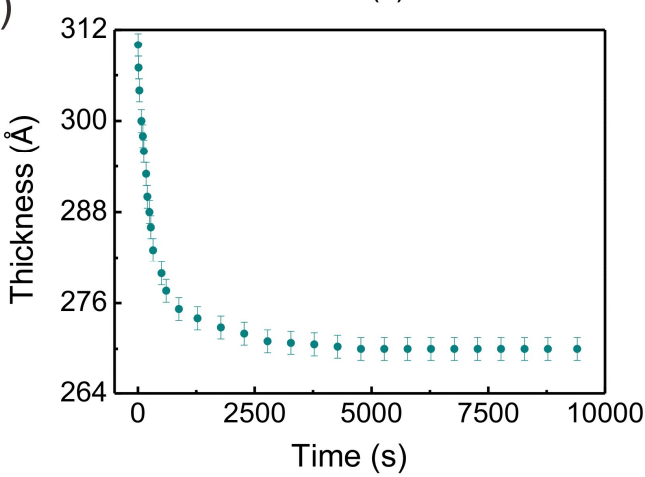

b)

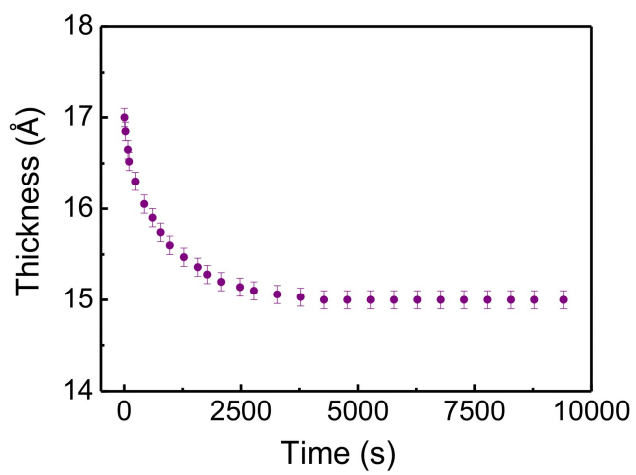

d)

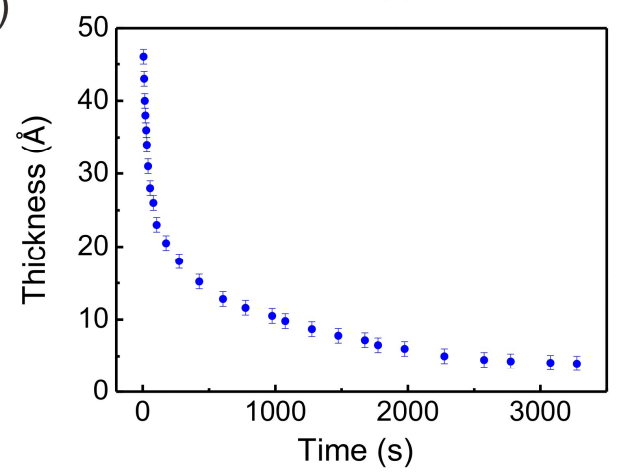

Figure S3. Thickness evolution in (a) PAN substrate coating (red), (b) substrate-near layer (purple), (c) main layer (dark green) and (d) vapor-near layer (blue) after the temperature is rapidly increased from 23 to $50^{\circ} \mathrm{C}$.

Table S2. Obtained fit parameters of vapor-near layer and main layer in the swollen film during dehydration (temperature increases from 23 to $50^{\circ} \mathrm{C}$ ).

\begin{tabular}{cccc}
\hline & $\tau / \mathrm{s}$ & $\mathrm{B}$ & $\varphi(\mathrm{D} 2 \mathrm{O})_{\infty} / \%$ \\
\hline vapor-near layer & $1000 \pm 20$ & $0.84 \pm 0.02$ & $3.7 \pm 0.5$ \\
main layer & $1800 \pm 20$ & $0.83 \pm 0.02$ & $2.2 \pm 0.5$ \\
\hline
\end{tabular}




\section{References}

1. Zhong, Q.; Metwalli, E.; Rawolle, M.; Kaune, G.; Bivigou-Koumba, A. M.; Laschewsky, A.; Papadakis, C. M.; Cubitt, R.; Wang, J. P.; Müller-Buschbaum, P. Influence of Hydrophobic Polystyrene Blocks on the Rehydration of Polystyreneblock-poly(methoxy diethylene glycol acrylate)-block-polystyrene Films Investigated by in situ Neutron Reflectivity. Macromolecules 2016, 49, 317-326.

2. Wang, W. N.; Metwalli, E.; Perlich, J.; Troll, K.; Papadakis, C. M.; Cubitt, R.; Müller-Buschbaum, P. Water Storage in Thin Films Maintaining the Total Film Thickness as Probed with in situ Neutron Reflectivity. Macromol. Rapid. Comm. 2009, 30, 114-119. 